\title{
Editorial
}

\section{Nanostructures for Flexible Electronics and Drug Delivery}

\author{
Chuanfei Guo, ${ }^{1}$ Jian Zhong, ${ }^{2}$ Liqian Gao, ${ }^{3}$ Jinwei Gao, ${ }^{4}$ and Siya Huang ${ }^{5}$ \\ ${ }^{1}$ Southern University of Science and Technology, Shenzhen, Guangdong 518055, China \\ ${ }^{2}$ Laboratory of Quality \& Safety Risk Assessment for Aquatic Products on Storage and Preservation (Shanghai), \\ Ministry of Agriculture, Shanghai Engineering Research Center of Aquatic-Product Processing \& Preservation, \\ College of Food Science \& Technology, Shanghai Ocean University, Shanghai 201306, China \\ ${ }^{3}$ School of Pharmaceutical Science (Shenzhen), Sun Yat-sen University (SYSU), Guangzhou, Guangdong 510275, China \\ ${ }^{4}$ Institute for Advanced Materials (IAM), Academy of Advanced Optoelectronics, South China Normal University, Guangzhou, China \\ ${ }^{5}$ Department of Physics and TcSUH, University of Houston, Houston, TX, 77204, USA
}

Correspondence should be addressed to Chuanfei Guo; guocf@sustc.edu.cn

Received 22 June 2017; Accepted 22 June 2017; Published 1 October 2017

Copyright (C) 2017 Chuanfei Guo et al. This is an open access article distributed under the Creative Commons Attribution License, which permits unrestricted use, distribution, and reproduction in any medium, provided the original work is properly cited.

Nanostructures, including metal nanowires, semiconducting nanowires, carbon nanotubes, and graphene sheets, have been widely used as the building blocks or drug carriers for different applications (such as flexible photoelectronics, eskins, soft machines, implantable electronics, drug delivery, health monitoring, and tissue engineering). In these applications, one of the emerging fields, flexible electronics, has attracted significant attention in both academic research and industrial applications during the past decade. Flexible electronic devices may also be implanted in the human body and combine with nanomedicine and drug delivery. Nanostructure based drug carriers allow for not only the delivery of small-molecule drugs, but also the delivery of peptides, nucleic acids, proteins, and so on. Targeting delivery of all these drug molecules to specific localization/area will reduce systemic side effects and allow for more efficient use of the drug. As fundamental research is called to fulfill the demands of industry and healthcare, the development of such nanotechnology and nanoscience has offered a good platform for flexible electronics and nanomedicine.

This special issue focuses on nanostructures for flexible electronics and their applications in drug delivery. This issue received seventeen submissions and finally accepted four exciting review papers and seven research papers. H. Lu et al. review the recent progress on nanostructures for drug delivery. T. Fan et al. summarize peptide self-assembled nanostructures for drug delivery applications. S. Pan et al. introduce the application of nanomaterials in stem cell regenerative medicine of orthopedic surgery. Z. Chen et al. highlight the advance of carbon nanotubes in cancer diagnostics and therapeutics. K. Sun et al. fabricate nanostructured surface with tunable contact angle hysteresis for in vitro cell cultures of different tumor cells. Y. Zhang et al. investigate the binding affinity, cellular uptake, and subsequent intracellular trafficking of the nanogene vector P123-PEI-R13. X. Tian et al. develop functional surface coating on cellulosic flexible substrates with improved water-resistant and antimicrobial properties by the use of $\mathrm{ZnO}$ nanoparticles. $\mathrm{Z}$. Fang et al. study dynamic uptake of free drug and nanostructures for drug delivery based on bioluminescence measurements. J. $\mathrm{Yu}$ et al. report optimal $\mathrm{Zn}$-modified $\mathrm{Ca}$-Si-based ceramic nanocoating with $\mathrm{Zn}$ ion release for osteoblast promotion and osteoclast inhibition in bone tissue engineering. $\mathrm{K}$. Yang et al. analyze the reaction and characterization of low-temperature effect of transition nanostructure metal codoped SCR catalyst. T. Wang et al. synthesize fluorescent carbon dots by gastrointestinal fluid treatment of Mongolia Har Gabur. Unfortunately, we received few submissions on flexible electronics, especially those combined with both flexible electronic platforms and drug delivery. Hopefully, this crossing field can attract more attention in the near future.

By compiling these papers, we hope that this special issue provides the readers with some valuable information 
and useful guides to the research field of nanostructures for flexible electronics and drug delivery.

\section{Chuanfei Guo \\ Jian Zhong \\ Liqian Gao \\ Jinwei Gao \\ Siya Huang}



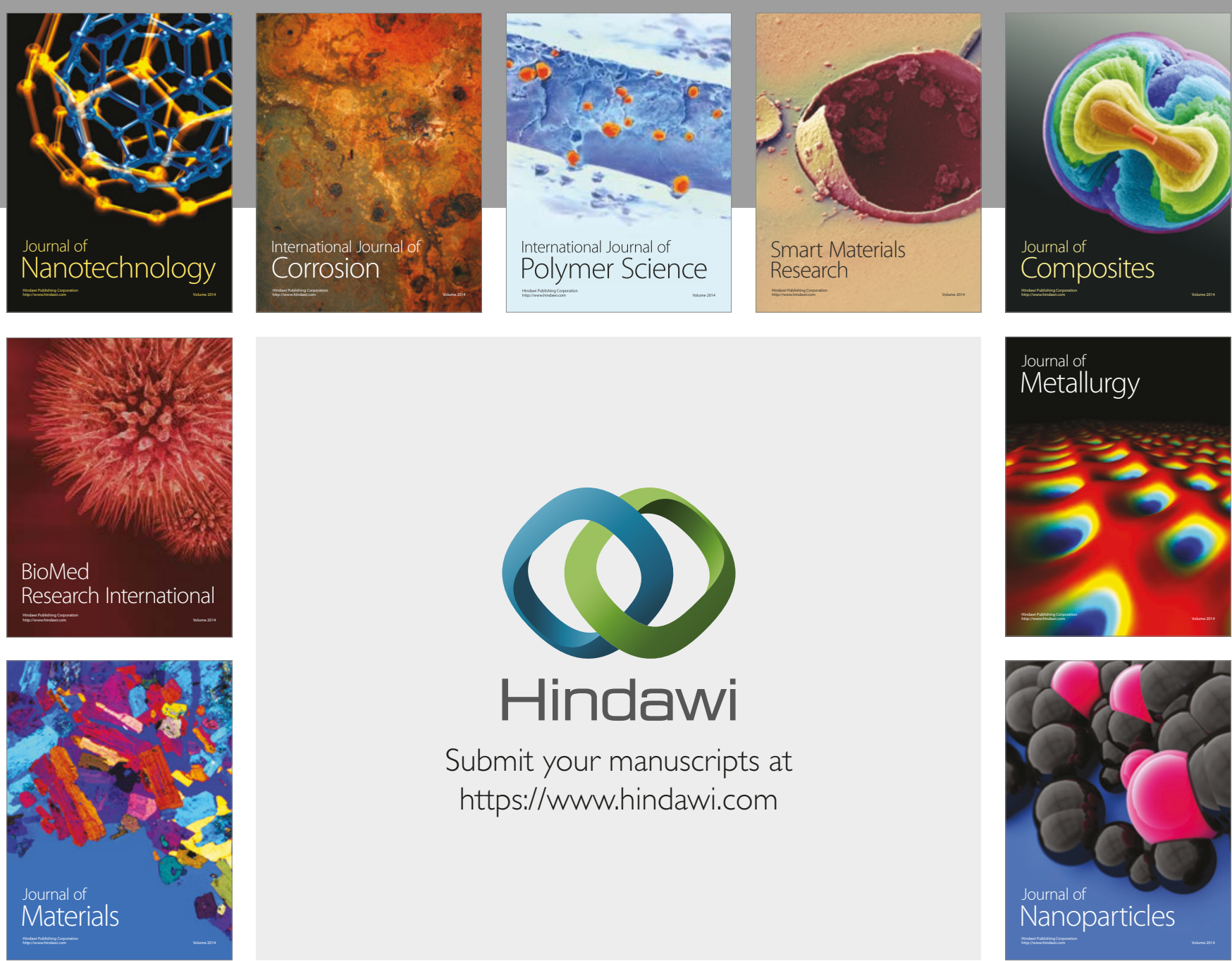

\section{Hindawi}

Submit your manuscripts at

https://www.hindawi.com
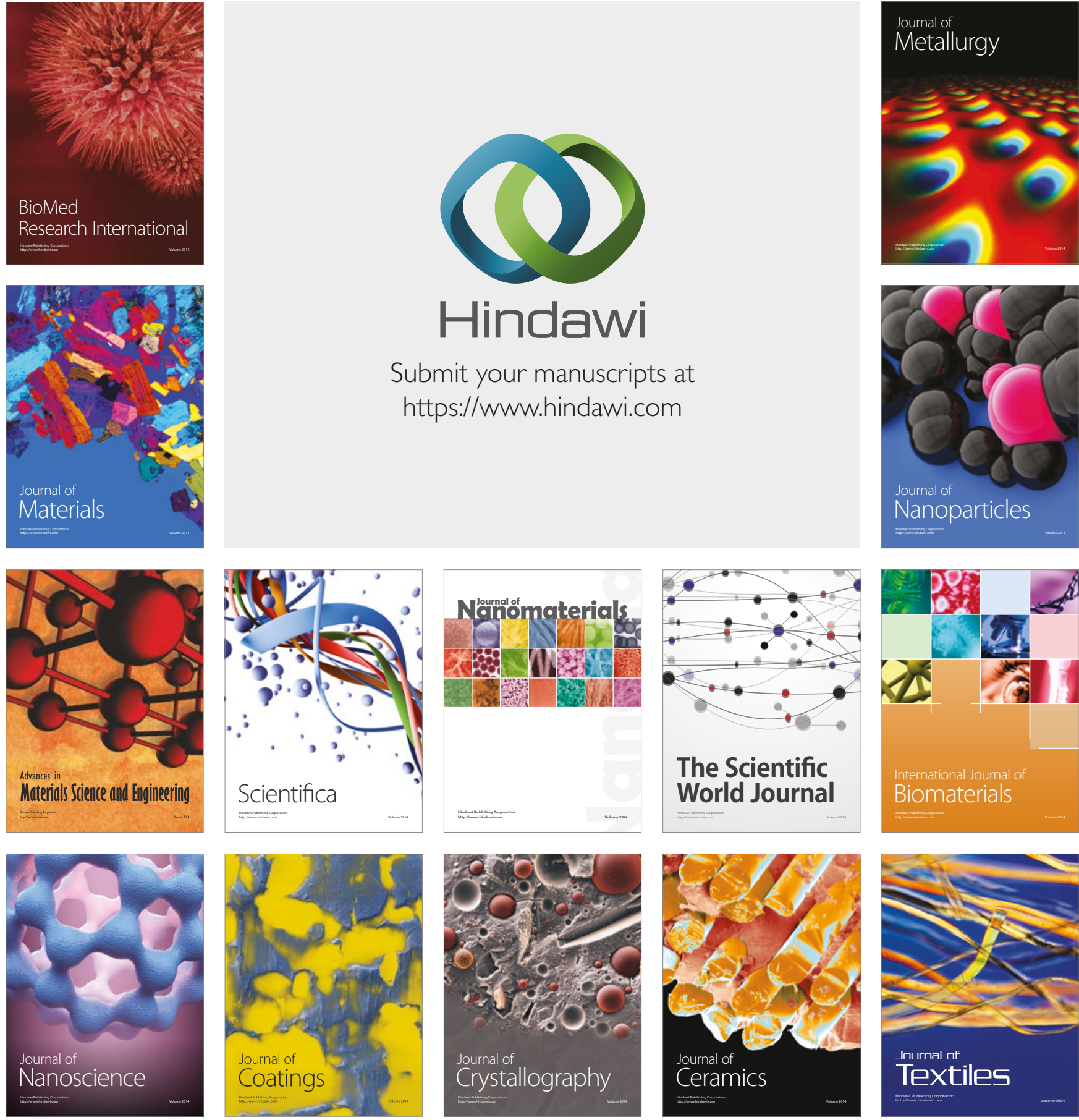

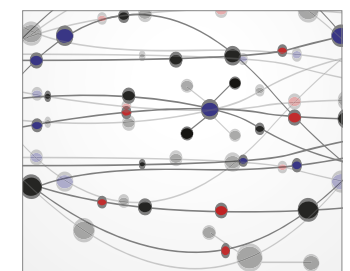

The Scientific World Journal
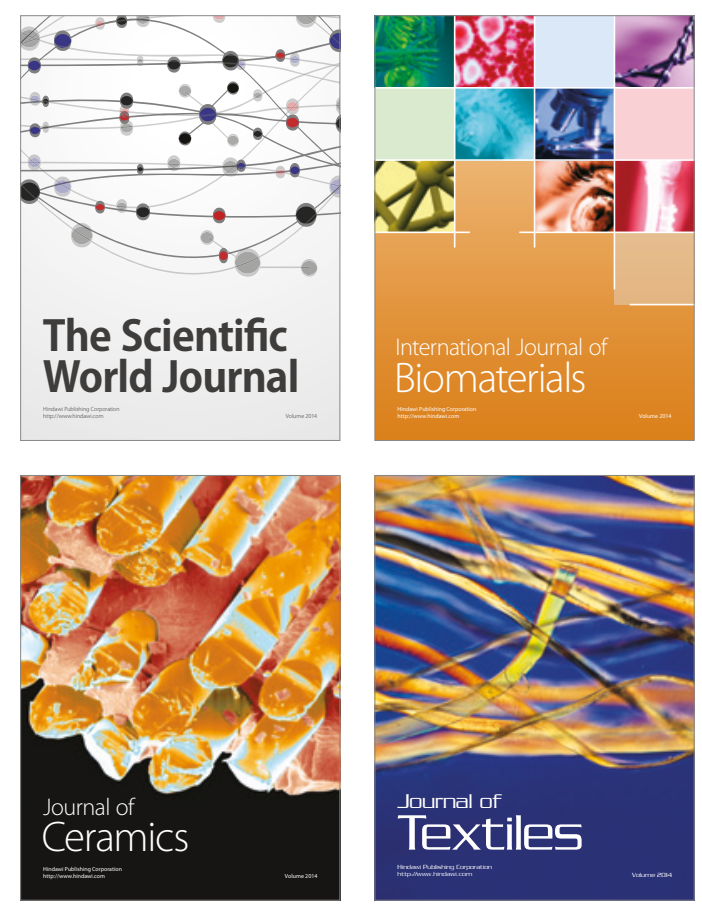